\title{
METODOLOGIAS ATIVAS NO ENSINO DE ENGENHARIA: ÊNFASE NA UTILIZAÇÃO DE MAPAS CONCEITUAIS NA APRENDIZAGEM SIGNIFICATIVA
}

\author{
ACTIVE METHODOLOGIES IN ENGINEERING TEACHING: EMPHASIS ON THE USE OF
} CONCEPTUAL MAPS IN SIGNIFICANT LEARNING

Betina Hansen ${ }^{1}$, Daniel Neutzling Lehn², Rafael Rodrigo Eckhardt ${ }^{3}$

DOI: 10.37702/REE2236-0158.v40p15-27.2021

\section{RESUMO}

O ano de 2020 ficará marcado pela pandemia da Covid-19, que exigiu um longo período de isolamento social e a incorporação de um conjunto de Tecnologias da Informação e Comunicação para manter as relações interpessoais, os estudos nos diferentes níveis da educação, o trabalho no modelo home office; além disso, os padrões de consumo foram impactados pela perda de empregos e a redução de renda. No ensino universitário emergiram e se fortaleceram as tecnologias digitais da informação e comunicação, servindo de meio e suporte para a educação não presencial, obrigando professores e estudantes a uma adaptação pessoal no processo de ensino e aprendizagem. O objetivo deste estudo foi avaliar a eficiência das metodologias ativas no ensino e na aprendizagem de estudantes de engenharia da Univates, englobando aulas presenciais e aulas não presenciais síncronas, com destaque para a incorporação de mapas conceituais nas aulas. A pesquisa consistiu em um projeto de intervenção pedagógica, mediada pelos três professores autores, realizada nos semestres de 2019B e 2020A e envolveu quatro turmas com estudantes dos cursos de Engenharia Civil, Engenharia Mecânica, Engenharia Ambiental e Engenharia Química. Foi utilizada a ficha de avaliação da intervenção pedagógica proposta por Almeida e Gomes (1997). Os resultados gerais do estudo evidenciaram que a utilização de mapas conceituais e outras metodologias ativas ampliaram o esforço e o trabalho pessoal dos estudantes nas aulas e evidenciaram uma melhora significativa na aprendizagem percebida pelos estudantes na comparação com aulas mais teóricas e conteudistas.

Palavras-chave: universidade; educação superior; metodologias ativas; mapas conceituais.

\begin{abstract}
The year 2020 will be marked by the Covid-19 pandemic, which required a long period of social isolation and the incorporation of a set of information and communication technologies to maintain interpersonal relationships, studies at different levels of education, work in the home office model and consumption patterns were impacted by the loss of jobs and the drop in income. In university education, digital information and communication technologies have emerged and strengthened, serving as a means and support for distance education, forcing teachers and students to personally adapt in the teaching and learning process. The aim of this study was to evaluate the efficiency of the active methodologies in teaching and learning of engineering students at Univates, encompassing face-to-face classes and synchronous distance classes, with emphasis on the incorporation of concept maps in classes. The research consisted of a pedagogical intervention project, mediated by the three author professors, carried out in the semesters of 2019B and 2020A and involved four classes with students from the civil engineering, mechanical engineering, environmental engineering and
\end{abstract}

\footnotetext{
${ }^{1}$ Professora, doutora em Engenharia e Ciência dos Materiais, Universidade do Vale do Taquari - Univates, betina.hansen@univates.br

${ }^{2}$ Professor, doutor em Engenharia e Ciência de Alimentos, Universidade do Vale do Taquari - Univates, lehn@univates.br

${ }_{3}^{3}$ Professor, mestre em Geoprocessamento e Sensoriamento Remoto, Universidade do Vale do Taquari - Univates, rafare@univates.br
} 
chemical engineering courses. To evaluate the pedagogical intervention, it was used an evaluation form proposed by Almeida and Gomes (1997). The general results of the study showed that the use of concept maps and other active methodologies increased the effort and personal work of students in classes and evidenced a significant improvement in the learning perceived by students in comparison with more theoretical and contented classes.

Keywords: university; college education; active methodologies; concept maps.

\section{INTRODUÇÃO}

A Portaria $\mathrm{n}^{\circ} 343$, de 17 de março de 2020 (BRASIL, 2020), autorizou às Instituições de Ensino Superior (IES) a substituição das aulas presenciais por aulas em meios digitais, enquanto durar a situação de pandemia. Nesse contexto, segundo Martins (2020), muitas barreiras que separavam a educação a distância da educação presencial foram transpostas.

Segundo a Unesco, mais de 1,3 bilhões de estudantes de todo o mundo foram afetados pelo fechamento das escolas e universidades devido ao necessário isolamento social para combater a pandemia da Covid-19. Esse número representa cerca de $80 \%$ da população estudantil mundial e afeta mais de 60 milhões de docentes, que não podem trabalhar de forma presencial, consolidando um desafio inédito à educação mundial. Para manter a relação com os estudantes e reduzir os prejuízos na aprendizagem, as instituições de ensino estão sendo obrigadas a inovar em suas metodologias de aprendizado virtual (SEMESP, 2020).

Considerando o referido cenário, as instituições privadas brasileiras de ensino superior, que são responsáveis por $75 \%$ dos estudantes de graduação, estão adotando novas plataformas de ensino e estão ampliando a segurança digital com a finalidade de ofertar um serviço remoto de qualidade para que seus estudantes não tenham prejuízo no ensino e na aprendizagem, além de contribuir na redução da inadimplência e no controle da evasão (SEMESP, 2020).

Nas Instituições de Ensino Superior do Comung, no estado do Rio Grande do Sul, de forma preponderante, o modelo de ensino adotado e praticado durante a pandemia foi o constituído por aulas síncronas, nomeadas como virtualizadas, remotas ou on-line, ou seja, professor e estudante estavam no mesmo ambiente (virtual), na mesma hora, de forma similar às aulas presenciais.

Diversas matérias de capa da Revista Ensino Superior, publicadas entre os anos de 2017 a 2020, anteriores à pandemia da Covid19, apontam para uma série de transformações educacionais contemporâneas, que envolvem o estudante, o professor, as estratégias e práticas pedagógicas, entre outros aspectos relacionados com o processo de ensino e de aprendizagem, que determinam a necessidade de aprimoramento das habilidades e competências didático-pedagógicas dos professores universitários no exercício da docência na contemporaneidade.

Os processos de ensino e de aprendizagem estão mais voltados ao que o mercado de trabalho exige dos profissionais na atualidade quando comparados ao passado, buscando favorecer o desenvolvimento de habilidades e competências cognitivas, procedimentais e atitudinais. Nesse processo são muito importantes as estratégias de ensino e aprendizagem escolhidos pelo professor e a participação ativa dos estudantes, seja em aulas presenciais ou em aulas virtualizadas.

Nessa questão reside um dos grandes desafios da docência universitária contemporânea: como conseguir ministrar aulas atrativas, utilizando metodologias ativas de ensino e de aprendizagem, por meio de aulas presenciais e de tecnologias da comunicação e informação, se a maioria das experiências dos professores na graduação, mestrado $e$ doutorado envolveram um processo de ensino tradicional, focado no conteúdo e pouco em aplicações na sociedade? Sabendo que a docência é uma responsabilidade complexa (DEWEY, 1971; MORIN, 2005), a resposta a 
esse desafio é a busca pela compreensão das bases da educação, a importância de humanizar a docência e a incorporação de novas metodologias e tecnologias pelos professores.

Moscovicci (2011) destaca que o processo de interação humana é permanente, complexo e desafiador, envolvendo comportamentos, emoções e manifestações verbais e não-verbais. Considerando que o processo de ensino e aprendizagem, em qualquer nível de educação, envolve a interação professor e estudante, é natural que a complexidade das interações humanas influencie nos processos pedagógicos e na aprendizagem dos estudantes, principalmente no ensino superior, no qual a maioria dos estudantes trabalham durante o dia e estudam à noite, além de terem uma série de compromissos financeiros, preocupações familiares, desejos materiais, necessidades de lazer e distrações diversas.

Larrosa (2018) enfatiza que a docência aposta nas maneiras de conceber uma aula, nas condições de criação, experimentação e reflexão docente, do professor como mediador do processo de aprendizagem, vislumbrando o crescimento do estudante. Cabe aos professores a importante tarefa de selecionar aquilo que vai ser disparador da atenção e dos pensamentos dos estudantes. Essas escolhas, assim como as estratégias de ensino, devem ser realizadas com sabedoria. Zabalza (2004) enfatiza que o professor é o elemento essencial que fundamenta a prática educativa, é quem faz a diferença em um cenário complexo e multidimensional como o atual, no qual entrecruzam influências dos mais diversos tipos. O professor mediador estimulará o estudante a pensar, refletir, se envolver e criar em desafios de aprendizagem, com forte conexão com problemas da sociedade e demandas do mercado de trabalho.

Um dos desafios contemporâneos consiste em fazer os processos de ensino acompanharem a velocidade dos avanços tecnológicos e as transformações da sociedade, com o objetivo de se obter efeitos positivos sobre a qualidade do ensino e da aprendizagem. Conforme Batalha (2018), a manutenção da motivação para a aprendizagem dos estudantes tem se tornado um desafio, fazendo com que o professor tenha que motivar os estudantes a partir da inovação em seu trabalho pedagógico, realizando-o com criatividade, reconhecendo o esforço dos estudantes e aproveitando as oportunidades de aprendizagem que surgem.

Independente da teoria educacional escolhida para explicar o processo de ensino e aprendizagem, professor e estudante têm um papel fundamental nesse processo. Os estudantes, como parte ativa na produção do seu conhecimento, e os docentes, como estimuladores desse processo. Para isso, os docentes buscam diferentes estratégias e tecnologias de ensino.

De acordo com Berbel (2011), as metodologias ativas envolvem uma concepção de ensino que utiliza experiências simuladas ou reais, com o objetivo de promover no estudante a habilidade de resolver problemas em diferentes contextos da sociedade ou da atuação profissional, proporcionando a formação de um indivíduo ativo, crítico, reflexivo e ético, por meio da aprendizagem significativa, além de exigir indivíduos conscientes e comprometidos com a qualidade de seu saber.

Segundo Demo (2018), o desafio contemporâneo do ensino superior é colocar o estudante no centro do processo de ensino e aprendizagem, estimulando aulas mais dinâmicas e ativas. Cabe aos estudantes ampliar a autonomia e o protagonismo na construção do seu conhecimento e reconhecer os benefícios de sua participação nesse processo.

Esse modelo de ensino superior contemporâneo está alinhado a uma Epistemologia Construtivista e a uma Pedagogia Relacional ou Interacionista (BECKER, 2012). Para que ocorra a promoção da autonomia na aprendizagem, de acordo com Berbel (2011), é necessário que o estudante tenha comprometimento pessoal, vivencie uma relação horizontal com seu professor e disponha de flexibilidade na execução das tarefas propostas.

São muitas as possibilidades de utilizar metodologias ativas no ensino dos cursos de graduação em Engenharia, sendo que o uso dessas práticas pedagógicas está sendo progressivamente ampliado, com potencial de utilização nas aulas presenciais e também on- 
line. Entre as estratégias e metodologias ativas do ensino superior destacam-se as seguintes: problematização; estudo de caso; seminários; aprendizagem baseada em problemas (Problem-Based Learning); aprendizagem baseada em projetos (Project Based Learning ); aprendizagem entre pares ou times (Team Based Learning); sala de aula invertida (Flipped Classroom); e mapas conceituais.

Entre as estratégias ou metodologias ativas utilizadas, destacam-se os mapas conceituais. A teoria que está por trás do mapeamento conceitual é a teoria cognitiva de aprendizagem de David Ausubel (AUSUBEL; NOVAK; HANESIAN, 1978; AUSUBEL, 2003; MOREIRA; MASINI, 2006; MOREIRA, 1999; VALADARES; MOREIRA, 2009). No entanto, trata-se de uma técnica desenvolvida por Joseph Novak e colaboradores na Universidade de Cornell (USA), em meados da década de 1970.

Segundo Tavares (2007), os mapas conceituais hierárquicos se apresentam como instrumentos adequados para estruturar um conhecimento em construção pelo estudante, da mesma forma que se mostra adequado para explicitar o conhecimento de um especialista, constituindo-se como um instrumento facilitador da aprendizagem, possibilitando ao estudante aprender a aprender com autonomia e independência. Os mapas conceituais foram desenvolvidos para promover a aprendizagem significativa.

Ao considerar que o estudante é construtor de seu conhecimento e o faz por meio de aprendizagem significativa, ocorre o estabelecimento de relações entre o que está aprendendo e o que já sabe, sendo a tarefa principal do professor elaborar estratégias que permitam o acesso aos conhecimentos tácitos, em que a ordenação e sequenciamento de conceitos e conhecimentos de modo hierárquico em classes e subclasses constituemse como recurso metodológico relevante ao compor ou organizar um mapa conceitual (CARABETTA JÚNIOR, 2013).

Os mapas conceituais, segundo Silva, Claro e Mendes (2017), são representações gráficas semelhantes a diagramas de fluxos, que indicam as relações entre conceitos, sendo utilizados para auxiliar na ordenação e na sequenciação hierarquizada dos conteúdos em determinada matéria de estudo, para oferecer estímulos adequados ao estudante. São como instrumentos para facilitar o aprendizado dos conteúdos sistematizados em aprendizagens significativas para o aprendiz.

Silva, Neves e Silva (2014) afirmam que mapas conceituais servem como eficientes meios de instrução e também como ferramentas de avaliação, quando se prioriza a evolução conceitual e reestruturação de conceitos e significados, elementos imprescindíveis no processo de ensino e aprendizagem significativa.

Correia et al. (2009) comentam que a incorporação dos mapas conceituais nas aulas exige uma abertura para a incerteza, pois eles permitem a explicitação das características comportamentais presentes nos complexos modelos mentais dos estudantes. Os autores indicam que no trabalho com mapas conceituais há o desafio de lidar com a subjetividade, mas oportunizam o diálogo e as interações entre pares (aluno/aluno) e entre professor/estudante. Um exemplo de mapa conceitual é mostrado na Figura 1.

Figura 1 - Esquematização de um mapa conceitual

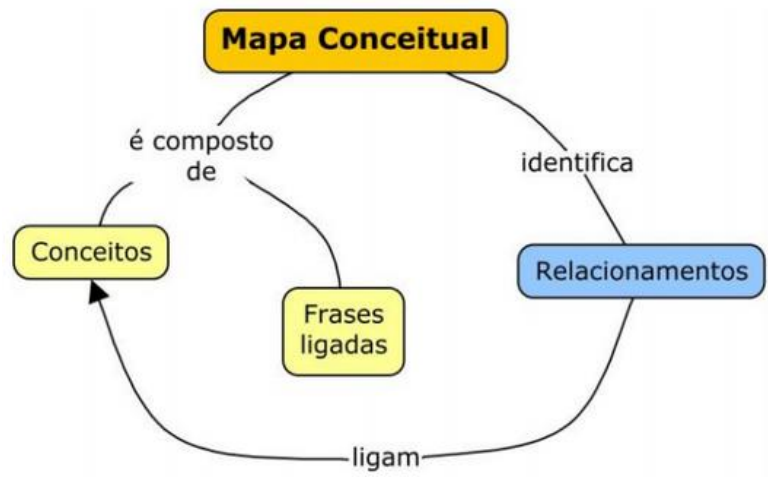

Fonte: Silva, Claro e Mendes (2017).

Dessa forma, neste trabalho foi analisada a utilização de metodologias ativas, em especial a incorporação dos mapas conceituais na aprendizagem significativa nas aulas de componentes curriculares da Engenharia da Univates, no contexto das aulas presenciais e virtualizadas síncronas, realizadas antes e durante a influência da pandemia da Covid-19. 


\section{METODOLOGIA}

Esta pesquisa, do tipo intervenção pedagógica, foi realizada no segundo semestre de 2019 e no primeiro semestre de 2020 com estudantes dos cursos de Engenharia Civil, Engenharia Mecânica, Engenharia Ambiental e Engenharia Química da Universidade do Vale do Taquari, localizada na cidade de Lajeado, no estado do Rio Grande do Sul. Os estudantes da Univates se caracterizam por trabalharem durante o dia e estudarem durante a noite, com tempo limitado para a dedicação extraclasse.

De acordo com Tripp (2005) e Thiollent (2009), as pesquisas do tipo intervenção pedagógica devem produzir mudanças, resolver um problema, ter caráter aplicado, necessitar de diálogo com referencial teórico e possibilitar a produção de conhecimento. De acordo com Gil (2010), um experimento consiste em definir um objeto de estudo, selecionar as variáveis capazes de influenciá-lo e definir as formas de controle e de observação dos efeitos que a variável produz no objeto. As intervenções pedagógicas envolvem planejamento e implementação de uma interferência e a avaliação de seus efeitos.

As intervenções pedagógicas foram aplicadas nas disciplinas de Conformação Mecânica (Engenharia Mecânica), Transferência de Calor e Massa (Engenharia Química e Engenharia Mecânica), Geoprocessamento e Sensoriamento Remoto e Topografia e Geoprocessamento (ambas da Engenharia Civil e Engenharia Ambiental).

A disciplina de Conformação Mecânica possui 60 horas distribuídas em encontros semanais e é uma disciplina do quinto semestre do curso de Engenharia Mecânica. Nela, os estudantes devem relacionar conceitos teóricos de metalurgia dos materiais aos diferentes métodos de conformação e seus respectivos cálculos de força, deformação, rendimento, etc. Ao ministrar a disciplina, o professor percebe dificuldades dos estudantes em relacionar esses conceitos teóricos à parte prática, motivados pela dificuldade de interpretar e de relacionar conceitos. Dessa forma, a utilização de mapas conceituais pode auxiliar nessa identificação e relação. No segundo semestre de 2019 havia 24 estudantes matriculados nessa disciplina, sendo que destes 19 participaram efetivamente da pesquisa.

A disciplina de Transferência de Calor e Massa $(60 \mathrm{~h})$ possui pré-requisitos, sendo eles as disciplinas de Fenômenos de Transporte e de Termodinâmica, para o curso de Engenharia Química; e as disciplinas de Mecânica dos Fluidos e Termodinâmica para a Engenharia Mecânica. O conteúdo da disciplina de Transferência de Calor e Massa é, muitas vezes, referido pelos estudantes como tendo um grau de complexidade e dificuldade que, por sua vez, gera uma preocupação excessiva e ansiedade, por exigir, em teoria, um tempo de dedicação maior em relação a outras disciplinas. $\mathrm{Na}$ condução da disciplina de Transferência de Calor e Massa, há alguns anos, percebe-se que a grande dificuldade dos estudantes não está nos conteúdos em si, mas na relação de conhecimentos anteriores (que devem ser estudados nos pré-requisitos) com os conceitos e aplicações executados na disciplina. Muitas vezes a falta de atenção ao executar análises de situações ou operações matemáticas simples e mais complexas acaba comprometendo o avanço dos estudantes no decorrer da disciplina. Por esses e outros motivos a disciplina tem apresentado reprovações em cerca de $25 \%$ da turma a cada semestre, tornando-se alvo de preconceitos equivocados em relação a tal disciplina por parte dos estudantes. No segundo semestre de 2019 essa disciplina contava com 28 estudantes matriculados.

As disciplinas de Geoprocessamento e Sensoriamento Remoto ( $60 \mathrm{~h}$ ) e de Topografia e Geoprocessamento $(80 \mathrm{~h})$ são componentes curriculares obrigatórios da Engenharia Civil e da Engenharia Ambiental, ocorrendo de forma integral em laboratórios de informática. As disciplinas têm uma proposta principal de desenvolver habilidades procedimentais, práticas e operacionais, envolvendo a manipulação de equipamentos tecnológicos, bases digitais de informações, imagens de satélite e softwares específicos, como Autocad, Idrisi e ArcGis, em demandas reais da sociedade e da atuação profissional. A maioria dos estudantes chega na disciplina com um base 
reduzida de manipulação de softwares. Nesse sentido, desafios de aprendizagem são conduzidos pelo professor como um guia que percorre as funções ordenadas nos programas, partindo de um problema real, mostrando os passos e as operações até alcançar o resultado. Essa dinâmica é alterada em algumas aulas com um tutorial passo a passo, em que os estudantes seguem as tarefas sem supervisão do professor. Em ambas as situações, quando os estudantes são desafiados a utilizar os recursos e os softwares para encontrar soluções utilizando a sua autonomia e independência, eles têm muitas dificuldades. A utilização de mapas conceituais e outras metodologias ativas pode auxiliar os estudantes a delinear as soluções aos desafios de aprendizagem, partindo-se da identificação dos recursos necessários (imagens de satélite, bases digitais de informações, softwares), dos procedimentos, até alcançar os resultados, por meio dos relacionamentos e das conexões. A disciplina de Topografia e Geoprocessamento teve 23 estudantes matriculados e de Geoprocessamento e Sensoriamento Remoto teve 30 estudantes matriculados no primeiro semestre de 2020. A intervenção nessas duas disciplinas ocorreu durante as aulas presenciais e, na sequência, durante as aulas virtualizadas síncronas, em razão da evolução da pandemia da Covid-19, que levou à suspensão das aulas presenciais a partir da metade do mês de março de 2020.

A intervenção se desenvolveu com base na comparação da autoavaliação dos estudantes após uma aula expositivo-dialogada e após uma aula cujo método aplicado foi a utilização de mapas conceituais ou outra metodologia ativa utilizada de forma complementar.

A metodologia de construção dos mapas conceituais foi exposta previamente para os estudantes, de modo a elencar os objetivos, as aprendizagens potenciais e permitir a organização operacional dos referidos mapas conceituais a partir de uma matéria em estudo. Nessa etapa foram elucidadas as dúvidas dos estudantes sobre como preparar os mapas, quais ferramentas tecnológicas estão disponíveis para a organização, entre outras dúvidas, pois os estudantes não tinham claros o conceito e $\mathrm{o}$ processo de organização de um mapa conceitual. Após, foi solicitado que os estudantes elaborassem de maneira individual ou em grupos um mapa conceitual de uma matéria em estudo em cada componente curricular. Como instrumento de avaliação da intervenção pedagógica realizada nas quatro disciplinas foi utilizada a Ficha de Avaliação de Aula, elaborada em escala numérica, por Almeida e Gomes (1997) (Figura 2).

Figura 2 - Ficha de Avaliação de Aula

P1. Meu estado de espírito quando cheguei hoje para a aula era:

\begin{tabular}{|l|l|l|l|l|l|l|l|l|l|l|}
\hline 0,0 & 0,5 & 1,0 & 1,5 & 2,0 & 2,5 & 3,0 & 3,5 & 4,0 & 4,5 & 5,0 \\
\hline
\end{tabular}

P2. Durante a aula posso dizer que me senti:

\begin{tabular}{|c|c|c|c|c|c|c|c|c|c|c|}
\hline 0,0 & 0,5 & 1,0 & 1,5 & 2,0 & 2,5 & 3,0 & 3,5 & 4,0 & 4,5 & 5,0 \\
\hline \multicolumn{11}{|c|}{ P3. $\mathrm{O}$ assunto desenvolvido na aula de hoje quanto à relevância para mim foi: } \\
\hline 0,0 & 0,5 & 1,0 & 1,5 & 2,0 & 2,5 & 3,0 & 3,5 & 4,0 & 4,5 & 5,0 \\
\hline \multicolumn{11}{|c|}{ P4. Considerando meu esforço pessoal, minha avaliação sobre meu trabalho na aula foi: } \\
\hline 0,0 & 0,5 & 1,0 & 1,5 & 2,0 & 2,5 & 3,0 & 3,5 & 4,0 & 4,5 & 5,0 \\
\hline \multicolumn{11}{|c|}{ P5. O resultado que alcancei na aula de hoje, quanto à aprendizagem foi: } \\
\hline 0,0 & 0,5 & 1,0 & 1,5 & 2,0 & 2,5 & 3,0 & 3,5 & 4,0 & 4,5 & 5,0 \\
\hline \multicolumn{11}{|c|}{ P6. Na minha opinião a atuação do professor para o meu aprendizado foi: } \\
\hline 0,0 & 0,5 & 1,0 & 1,5 & 2,0 & 2,5 & 3,0 & 3,5 & 4,0 & 4,5 & 5,0 \\
\hline \multicolumn{11}{|c|}{ P7. A atuação da turma para o meu desenvolvimento pessoal e profissional foi: } \\
\hline 0,0 & 0,5 & 1,0 & 1,5 & 2,0 & 2,5 & 3,0 & 3,5 & 4,0 & 4,5 & 5,0 \\
\hline
\end{tabular}

Fonte: Almeida e Gomes (1997).

Com o preenchimento da Ficha de Avaliação da Aula pelos estudantes, em cada intervenção pedagógica realizada, foi possível realizar comparações entre as diferentes aulas 
da mesma disciplina, bem como a comparação entre as percepções dos estudantes nas diferentes disciplinas e cursos de engenharia envolvidos, de modo a ressaltar os aspectos positivos e negativos da aplicação das metodologias ativas, em especial para os mapas conceituais.

Almeida e Gomes (1997) afirmam que a utilização de novos e eficientes métodos de ensino amplia a atuação do professor para atender estudantes com qualidade e se referem a três princípios básicos para $\mathrm{o}$ desenvolvimento do ensino e aprendizagem, sendo eles: criar um ambiente afetivo de apoio e de confiança em sala de aula; explicitar em todos os encontros a relevância do assunto abordado para a formação e vida dos estudantes; e, por fim, o incentivo à auto avaliação. No trabalho os autores apresentam a "Ficha de Avaliação de Aula" constituída por sete perguntas criadas com a finalidade de abranger os três princípios básicos citados.

\section{RESULTADOS E DISCUSSÕES}

Para a disciplina de Transferência de Calor e Massa (2019B), a Tabela 1 apresenta os resultados médios dos questionários aplicados. A partir da comparação das médias das respostas dos 42 estudantes da disciplina, é possível traçar algumas discussões. A maior diferença encontrada está na pergunta 7 , sobre a atuação da turma para o desenvolvimento pessoal, pois na construção coletiva do mapa conceitual, fica evidente a necessidade de participação dos estudantes que estão conscientes da proposta do estudo prévio do conteúdo para que possam contribuir para a construção do mapa da turma. Dessa forma, o estudante percebe seu protagonismo e de seus colegas para uma construção coletiva, na qual o resultado é compartilhado com todos.

Tabela 1 - Médias das respostas dos estudantes na avaliação do projeto de intervenção envolvendo uso de mapa conceitual para a disciplina de Transferência de Calor e Massa $(n=42)$

\begin{tabular}{|c|c|c|}
\hline Pergunta & $\begin{array}{l}\text { Média das respostas }( \pm \mathrm{DP}) \\
\text { Sem mapa conceitual }\end{array}$ & $\begin{array}{l}\text { Média das respostas }( \pm D P) \\
\text { Com mapa conceitual }\end{array}$ \\
\hline P1 (estado de espírito) & $\mathbf{3 , 6 8} \pm 0,77$ & $\mathbf{3 , 3 8} \pm 1,27$ \\
\hline P2 (sentimento na aula) & $\mathbf{4 , 0 0} \pm 0,57$ & $\mathbf{3 , 6 6} \pm 1,22$ \\
\hline P3 (relevância do assunto) & $4,47 \pm 0,46$ & $\mathbf{4 , 5 4} \pm 0,52$ \\
\hline P4 (esforço pessoal) & $\mathbf{3 , 7 6} \pm 0,85$ & $\mathbf{3 , 8 9} \pm 0,88$ \\
\hline P5 (resultado na aprendizagem) & $4,02 \pm 0,69$ & $\mathbf{4 , 2 5} \pm 0,83$ \\
\hline P6 (atuação do professor) & $\mathbf{4 , 5 6} \pm 0,43$ & $4,50 \pm 0,69$ \\
\hline P7 (atuação da turma) & $3,47 \pm 1,08$ & $4,27 \pm 0,60$ \\
\hline
\end{tabular}

Fonte: elaborada pelos autores.

A segunda maior diferença entre os resultados está na pergunta 2 , que se refere ao sentimento durante a aula, na qual o aluno expõe a redução do valor da resposta na aula com o uso do mapa conceitual. Justificando, o cansaço dos estudantes ao enfrentarem uma aula noturna depois de uma jornada de trabalho (estudante trabalhador) pode ser indicado como uma causa dessa variação, uma vez que, para participar da construção do mapa, os estudantes devem se apropriar dos conceitos do conteúdo, por vezes relacionando-o com conteúdos anteriores de outras disciplinas, o que exige tempo e dedicação. Mesmo com maior 
envolvimento e percebendo o resultado de aprendizagem como melhor (P5), os estudantes se sentem mais exigidos, ao estado de espírito, fator em princípio não determinado por ações da disciplina, exceto na situação de alguma discordância que possa ocorrer entre a turma e o docente nos encontros anteriores à aplicação da pesquisa, fato que não ocorreu na coleta de dados desta turma.

Para a disciplina de Conformação Mecânica (2019B), a Tabela 2 apresenta os resultados médios dos questionários de avaliação aplicados.

Tabela 2 - Médias das respostas dos estudantes na avaliação do projeto de intervenção envolvendo uso de mapa conceitual para a disciplina de Conformação Mecânica $(n=19)$

\begin{tabular}{lcc}
\hline \multicolumn{1}{c}{ Pergunta } & $\begin{array}{c}\text { Média das respostas ( } \begin{array}{c}\text { Sem mapa conceitual } \\
\text { Sem }\end{array} \\
\text { P1 (estado de espírito) }\end{array}$ & $\begin{array}{c}\text { Média das respostas ( } \pm \text { DP) } \\
\text { Com mapa conceitual }\end{array}$ \\
P2 (sentimento na aula) & $4,18 \pm 0,58$ & $3,42 \pm 0,93$ \\
P3 (relevância do assunto) & $4,29 \pm 0,54$ & $3,79 \pm 0,48$ \\
P4 (esforço pessoal) & $4,32 \pm 0,45$ & $4,16 \pm 0,58$ \\
P5 (resultado aprendizagem) & $4,34 \pm 0,58$ & $3,87 \pm 0,44$ \\
P6 (atuação do professor) & $4,39 \pm 0,52$ & $4,05 \pm 0,57$ \\
P7 (atuação da turma) & $4,61 \pm 0,46$ & $4,45 \pm 0,37$ \\
\hline
\end{tabular}

Fonte: elaborada pelos autores.

Ao analisar a Tabela 2 percebe-se um decréscimo em todas as avaliações realizadas pelos estudantes na aula em que foi aplicado o mapa conceitual, com as seguintes justificativas. Primeiramente, pode-se perceber, através dos resultados das perguntas P1 e P2, que os estudantes já iniciaram a aula com um estado de espírito inferior, bem como o seu sentimento durante a aula também está com uma avaliação inferior em relação à aula sem aplicação do mapa conceitual.

Além disso, através da pergunta P3 percebe-se que os estudantes avaliaram como menor a relevância do assunto abordado na aula em que o mapa conceitual foi aplicado. Isso se deve ao fato de que esta aula foi basicamente conceitual e teórica, abordando conceitos de metalurgia e de ciência dos materiais, os quais os estudantes já haviam aprendido em disciplinas anteriores; porém, agora, aplicados à conformação dos metais. Já a aula expositivadialogada abordou a parte inicial dos cálculos de conformação mecânica, sendo que a professora resolvia exercícios no quadro e, na sequência, os estudantes resolviam novos exercícios e eram discutidas as respostas. Provavelmente essa aula teve uma avaliação mais positiva justamente por ser mais prática e envolver cálculos. Já a aula teórica e conceitual envolve mais leitura e interpretação de texto, que são atividades não muito aceitas pelos estudantes de engenharia.

Pelo mesmo motivo justifica-se a avaliação das questões $\mathrm{P} 4$ a P7. Os estudantes perceberam um maior esforço pessoal na aula expositivadialogada por esta ter a resolução de exercícios, 
que é uma atividade que os estudantes consideram mais relevante do que a criação do mapa conceitual, resultando em maior aprendizagem (pergunta P5). Avaliaram também como maior a atuação da professora e da turma na aula expositiva-dialogada, devido à explicação teórica do conteúdo com resolução de exercícios.

As Tabelas 3 e 4 apresentam a avaliação das estratégias de ensino, respectivamente, das disciplinas de Geoprocessamento e Sensoriamento Remoto (GSR) e Topografia e Geoprocessamento (TG), ambas em 2020A. Ao analisar as tabelas, percebe-se que o estado de espírito dos estudantes ao chegar para a aula (P1) não alcança o indicador 4 em nenhuma das cinco autoavaliações realizadas, somado a um DP muito elevado. Seria adequado investigar melhor tal indicador, mas ele evidencia algum nível de falta de motivação inicial com relação às aulas. Esaa condição inicial é revertida no transcorrer da aula e os estudantes se sentiram (P2) melhor em aulas que utilizaram desafios práticos e operacionais de aprendizagem e também houve boa avaliação nas aulas que utilizaram mapas conceituais. As autoavaliações dos estudantes não apontaram diferenças significativas na percepção da relevância nos assuntos desenvolvidos (P3) considerando as diferentes estratégias de ensino utilizadas e nem na atuação da turma para o desenvolvimento pessoal e profissional (P7). A avaliação da atuação docente também é muito positiva independente da estratégia de ensino (P6).

Tabela 3 - Médias das respostas dos estudantes na avaliação do projeto de intervenção com uso de diferentes metodologias ativas na disciplina de Geoprocessamento e Sensoriamento Remoto $(n=25)$

\begin{tabular}{|c|c|c|c|}
\hline \multirow[b]{2}{*}{ Pergunta } & \multicolumn{3}{|c|}{ Média das respostas $( \pm \mathrm{DP})$} \\
\hline & $\begin{array}{c}\text { Introdução + } \\
\text { Aula Dialogada + } \\
\text { Mapa Conceitual }\end{array}$ & $\begin{array}{c}\text { Revisão + Aula } \\
\text { no Quadro + } \\
\text { Aplicação Prática }\end{array}$ & $\begin{array}{c}\text { Desafio de } \\
\text { Aprendizagem } \\
\text { Prático* }\end{array}$ \\
\hline P1 (estado de espírito) & $3,72 \pm 0,83$ & $3,62 \pm 1,07$ & $3,62 \pm 1,05$ \\
\hline P2 (sentimento na aula) & $3,98 \pm 0,69$ & $3,94 \pm 0,93$ & $4,31 \pm 0,65$ \\
\hline P3 (relevância do assunto) & $4,62 \pm 0,41$ & $4,52 \pm 0,62$ & $4,40 \pm 0,65$ \\
\hline P4 (esforço pessoal) & $4,10 \pm 0,66$ & $4,22 \pm 0,78$ & $4,45 \pm 0,62$ \\
\hline P5 (resultado na aprendizagem) & $4,32 \pm 0,51$ & $4,38 \pm 0,71$ & $4,52 \pm 0,57$ \\
\hline P6 (atuação do professor) & $4,72 \pm 0,43$ & $4,84 \pm 0,34$ & $4,81 \pm 0,36$ \\
\hline P7 (atuação da turma) & $4,18 \pm 0,84$ & $4,20 \pm 0,86$ & $4,07 \pm 1,20$ \\
\hline
\end{tabular}

\footnotetext{
* Aula realizada de forma virtualizada, utilizando as tecnologias da informação e comunicação em função da pandemia do Covid-19.

Fonte: elaborada pelos autores.
} 
Tabela 4 - Médias das respostas dos estudantes na avaliação do projeto de intervenção envolvendo uso de mapa conceitual para a disciplina de Topografia e Geoprocessamento $(\mathbf{n}=\mathbf{2 3})$

\begin{tabular}{|c|c|c|}
\hline Pergunta & $\begin{array}{l}\text { Média das respostas }( \pm \mathrm{DP}) \\
\text { Sem mapa conceitual }\end{array}$ & $\begin{array}{c}\text { Média das respostas }( \pm \mathrm{DP}) \\
\text { Com mapa conceitual }\end{array}$ \\
\hline P1 (estado de espírito) & $3,63 \pm 1,16$ & $3,97 \pm 0,94$ \\
\hline P2 (sentimento na aula) & $3,91 \pm 0,88$ & $4,13 \pm 0,78$ \\
\hline P3 (relevância do assunto) & $4,39 \pm 0,57$ & $4,50 \pm 0,74$ \\
\hline P4 (esforço pessoal) & $3,74 \pm 0,93$ & $4,21 \pm 0,71$ \\
\hline P5 (resultado na aprendizagem) & $4,09 \pm 0,64$ & $4,13 \pm 0,65$ \\
\hline P6 (atuação do professor) & $4,67 \pm 0,35$ & $4,68 \pm 0,40$ \\
\hline P7 (atuação da turma) & $4,46 \pm 0,62$ & $4,11 \pm 1,24$ \\
\hline
\end{tabular}

Fonte: elaborada pelos autores

Os resultados mais importantes na autoavaliação sobre as metodologias ativas implementadas pelo projeto de intervenção nas aulas das turmas de GSR (Tabela 3) e TG (Tabela 4) estão relacionados com a alteração do esforço e o trabalho pessoal dos estudantes (P4) e melhoria na percepção da aprendizagem alcançada pelos estudantes (P5). Ao utilizar metodologias ativas, como os mapas conceituais, aplicações práticas com softwares e desafios de aprendizagem conectados com as demandas da sociedade e relacionados com a atuação profissional na engenharia, os estudantes reconhecem necessidade de maior esforço e trabalho pessoal, mas com resultados positivos na aprendizagem significativa, principalmente na disciplina de GSR.

Cabe destacar que a melhor avaliação realizada pelos estudantes na disciplina de GSR para a atuação docente $(4,81)$, o sentimento positivo na aula $(4,13)$, o reconhecimento do esforço pessoal $(4,45)$ e a percepção de melhoria na aprendizagem $(4,52)$ ocorreu na primeira aula virtualizada da disciplina, na qual houve redução da aula teórica - dialogada - e a ampliação do uso de desafios práticos e operacionais de aprendizagem, em que os estudantes são desafiados a utilizarem os seus recursos tecnológicos, seus softwares, suas bases de informações, seus conhecimentos para gerar resultados relacionados com a atuação profissional.
As aulas subsequentes de GSR, que ocorreram no modelo virtualizado síncrono até a conclusão do semestre em função da pandemia, apesar de não terem sido avaliadas no âmbito da presente pesquisa, mantendo desafios práticos e operacionais de aprendizagem, mantiveram alta satisfação dos estudantes pela percepção de estarem aprendendo com autonomia e independência.

\section{CONCLUSÕES}

Os resultados derivados do projeto de intervenção pedagógica implementado com quatro turmas da engenharia da Univates, no segundo semestre de 2019 e primeiro semestre de 2020, antes e durante a pandemia da Covid19, permitiram verificar a importância do estado de espírito dos estudantes ao ir para as aulas, bem como o sentimento dos estudantes durante as aulas, a relevância do assunto para eles, o esforço pessoal durante a aula, a atuação do professor para o aprendizado, a atuação da turma para o desenvolvimento dos colegas e os resultados alcançados pelos estudantes em termos de aprendizagem significativa.

Em uma escala de 1 a 5, considerando as médias de todas as sete avaliações realizadas, $\mathrm{o}$ estado de espírito dos estudantes ao irem para as aulas (P1) supera o indicador 4 somente uma vez. Esse indicador deve ser mais bem 
explorado em pesquisas futuras, mas aponta para uma falta de motivação inicial dos estudantes ao irem para as aulas, podendo isso ter relação com a condição de estudante trabalhador. Esta condição inicial é revertida no transcorrer das aulas nas disciplinas de Geoprocessamento e Sensoriamento Remoto e de Topografia e Geoprocessamento, que utilizaram metodologias ativas diversificadas e desafios práticos de aprendizagem na sequência do semestre, estimulando os estudantes a se sentissem melhor durante as aulas (P2). Nas disciplinas de Transferência de Calor e Massa e de Conformação Mecânica, o sentimento dos estudantes até reduziu nas aulas que incorporaram mapas conceituais como metodologias ativas na comparação com aulas mais tradicionais.

Os estudantes, com exceção da turma de Conformação Mecânica, entenderam que a utilização de mapa conceitual nas aulas exigiu mais esforço e trabalho pessoal (P4), resultando em melhoria também na aprendizagem (P5). Essa percepção ficou ainda mais clara na disciplina de Geoprocessamento e Sensoriamento Remoto, na primeira aula virtualizada em função da pandemia, quando a estratégia da aula envolveu um desafio prático e operacional de aprendizagem. Os estudantes pontuaram muito bem a atuação docente, a percepção do esforço pessoal e de aprendizagem significativa construída com autonomia e independência. A autonomia e a independência dos estudantes acabam sendo atributos muito importantes na nova modalidade de ensino que está se fortalecendo no Brasil e no mundo em função da pandemia.

Por fim, conclui-se que a utilização de mapas conceituais, somada com outras metodologias ativas e desafios práticos de aprendizagem, estimula a percepção dos estudantes de coparticipação no processo de ensino e aprendizagem e de melhoria na aprendizagem na comparação com aulas mais teóricas e conteudistas.

\section{REFERÊNCIAS}

ALMEIDA, T. L.; GOMES, L. V. N. Aplicações de estatística multivariada na qualidade em sala de aula. Anais... Encontro Nacional de Engenharia de produção - ENEGEP, 1997.

AUSUBEL, D. P.; NOVAK, J. D.; HANESIAN, H. Educational psychology. New York: Holt, Rinehart and Winston, 1978.

AUSUBEL, D. P. Aquisição e retenção de conhecimentos: uma perspectiva cognitiva. Lisboa: Plátano Edições Técnicas, 2003.

BATALHA, C. E. A motivação para a aprendizagem significativa do aluno de ensino médio. Revista Multidisciplinar do Nordeste Mineiro - Unipac. p. 368-377, 2018.

BRASIL. Portaria n ${ }^{\circ}$ 343, de 17 de março de 2020. Disponível em: http://www.in.gov.br/en/web/dou/-/portaria-n343-de-17-de-marco-de-2020-248564376. Acesso em: 29 mai. 2020.

BECKER, F. Educação e construção do conhecimento. 2.ed. Porto Alegre: Penso, 2012.

BERBEL, N. A. N. As Metodologias ativas e a promoção da autonomia dos estudantes. Semina: Ciências Sociais e Humanas, Londrina, v. 32, n. 1, p. 25-40, jan./jun. 2011.

CARABETTA JÚNIOR, V. A utilização de mapas conceituais como recurso didático para a construção e inter-relação de conceitos. Revista Brasileira de Educação Médica, v. 37, n. 3, p. 441-447, 2013.

CORREIA, P. R. M. et al. Mapas conceituais como ferramenta de avaliação: desafios e possibilidades de mudanças na sala de aula. VII Encontro Nacional de Pesquisas em Educação em Ciências, 2009.

DEMO, P. Aula, meu xodó! Arquivo online disponível em: https://docs.google.com/ document/u/2/d/e/2PACX-

1vRaRIZKbxBHxQNILf2bucbzBLNryTfITG A410xHom0mbOlwIh1ooUWm3kSiKIOQkN FkZMSosdP8HJv7/pub, 2018. Acesso em: 8 ago. 2020.

DEWEY, J. Experiência e educação. Trad. Anísio Teixeira. São Paulo: Editora Nacional, 1971. 
GIL, A. C. Como Elaborar Projetos de Pesquisa.

5ªd. São Paulo: Atlas, 2010.

LARROSA, J. Esperando não se sabe o quê. Sobre o ofício de professor. São Paulo: Autêntica, 2018.

MARTINS, R. X. A Covid-19 e o fim da educação a distância: Um ensaio. EmRede, Porto Alegre, v. 7, n. 1, p. 242-256, jan./jun. 2020.

MOREIRA, M. A. Aprendizagem significativa. Brasília: Editora da UnB, 1999.

MOREIRA, M. A; MASINI, E. F. S. Aprendizagem significativa: a teoria de aprendizagem de David Ausubel. 2 ed. São Paulo: Centauro Editora, 2006.

MORIN, E. Os sete saberes necessários à educação do futuro. 10. ed. São Paulo: Cortez, Brasília, 2005.

MOSCOVICCI, F. Desenvolvimento Interpessoal: treinamento em grupo. 20a ed. Rio de Janeiro: José Olympio, 2011.

SEMESP. Efeitos da Pandemia na Educação Superior Brasileira. São Paulo: Instituto Semesp. Arquivo online disponível em: $\quad$ https://www.semesp.org.br/wpcontent/uploads/2020/04/estudo-0904.pdf.

Acesso em: 8 ago. 2020.
SILVA, W.; CLARO, G. R. C.; MENDES, A. P. Aprendizagem significativa e mapas conceituais. Anais... XIII Congresso Nacional de Educação, Curitiba, 2017.

SILVA, P. F. Z.O.; NEVES, M. C. D.; SILVA, S. C. R. Análise de mapas conceituais: uma perspectiva fenomenológica Aprendizagem Significativa em Revista, v.4, n. 3, p. 1-10, 2014.

TAVARES, R. Construindo mapas conceituais. Ciências \& Cognição, v. 12, p. 72-85, 2007.

THIOLLENT, M. Metodologia da Pesquisa-ação. 17 . ed. São Paulo: Cortez, 2009.

TRIPP, D. Pesquisa-ação: uma introdução metodológica. Educação \& Pesquisa, São Paulo, v. 31, n. 3, p. 443-466, set./dez. 2005.

VALADARES, J. A.; MOREIRA, M. A. A teoria da aprendizagem significativa. Sua fundamentação e implementação. Coimbra: Almedina, 2009.

ZABALZA, M. A. O ensino universitário: seu cenário e seus protagonistas. Porto Alegre: Artmed, 2004.

\section{DADOS BIOGRÁFICOS DOS AUTORES}

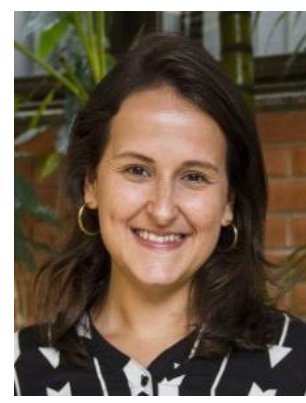

Betina Hansen - possui graduação em Engenharia de Materiais pela Universidade Federal do Rio Grande do Sul (UFRGS) e mestrado e doutorado em Ciência e Tecnologia dos Materiais pela Universidade Federal do Rio Grande do Sul (UFRGS). Atualmente é professora e coordenadora do curso de Engenharia Civil na Universidade do Vale do Taquari, em Lajeado/RS. Tem experiência na área de ciência dos materiais, materiais de construção civil, caracterização de materiais poliméricos e materiais compósitos com matriz termoplástica. 


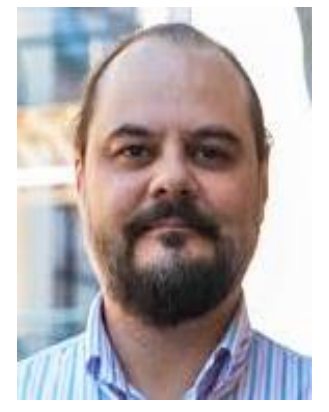

Daniel Neutzling Lehn - possui graduação em Engenharia de Alimentos (1996 FURG), mestrado e doutorado em Engenharia e Ciência de Alimentos (2018 FURG) e especialização em Gestão Universitária (2012 - UNIVATES). Atualmente é professor adjunto na Universidade do Vale do Taquari - Univates, em Lajeado/RS. Tem experiência nas áreas de tecnologia de alimentos e laticínios.

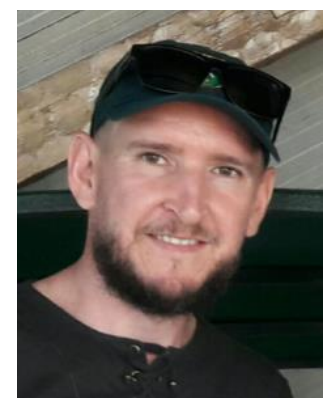

Rafael Rodrigo Eckhardt - possui graduação em Ciências Biológicas pela Universidade do Vale do Taquari (UNIVATES) e mestrado Geoprocessamento e Sensoriamento Remoto pela Universidade Federal do Rio Grande do Sul (UFRGS). Atualmente é professor e coordenador dos cursos Ciências Biológicas na Universidade do Vale do Taquari, em Lajeado/RS. Tem experiência nas áreas de geoprocessamento, sensoriamento remoto, topografia, gestão ambiental, desastres naturais e na elaboração e execução de projetos ambientais. 